\title{
The ERS-endorsed official ATS/CDC/IDSA clinical practice guidelines on treatment of drug-susceptible tuberculosis
}

\author{
Giovanni Sotgiu (1) 1, Payam Nahid², Robert Loddenkemper³ ${ }^{3}$ Ibrahim Abubakar ${ }^{4}$, \\ Marc Miravitlles ${ }^{5}$ and Giovanni Battista Migliori $\mathbb{1}^{6}$
}

Affiliations: ${ }^{1}$ Clinical Epidemiology and Medical Statistics Unit, Dept of Biomedical Sciences - University of Sassari - Research, Medical Education and Professional Development Unit, AOU Sassari, Sassari, Italy. ${ }^{2}$ University of California, San Francisco, CA, USA. ${ }^{3}$ German Central Committee against Tuberculosis (DZK), Berlin, Germany. ${ }^{4}$ Institute for Global Health, University College London, London, UK. ${ }^{5}$ Pneumology Dept, Hospital Universitari Vall d'Hebron, CIBER de Enfermedades Respiratorias (CIBERES), Barcelona, Spain. ${ }^{6}$ World Health Organization Collaborating Centre for Tuberculosis and Lung Diseases, Fondazione S. Maugeri, Care and Research Institute, Tradate, Italy.

Correspondence: Giovanni Battista Migliori, WHO Collaborating Centre for TB and Lung Diseases, Fondazione S. Maugeri, Care and Research Institute, via Roncaccio 16, 21049 Tradate, Italy.

E-mail: giovannibattista.miglioriafsm.it

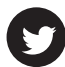

@ERSpublications

Updated clinical practice guidelines on TB treatment provide clinical and public health management recommendations http://ow.ly/FDcN302Jdmp

\section{Introduction}

The World Health Organization (WHO) estimates that 9.6 million cases of tuberculosis (TB) occurred in 2014 (corresponding to 133 cases per 100000 population) with 1.5 million deaths [1]. Furthermore, 3.3\% of new and $20 \%$ of previously treated cases harbour multidrug-resistant (MDR)-TB strains. Eastern European and central Asian countries still have the highest prevalence of MDR-TB. In low TB incidence countries (largely covering North America and Western Europe; figure 1) 155000 TB cases occur every year with over 10000 deaths [2].

Rapid diagnosis and effective treatment of newly diagnosed TB cases, the majority of whom are susceptible to first-line anti-TB drugs, constitutes the essence of TB control by curing the patient of TB and rapidly halting further transmission of in the community [3]. It is widely recognised that MDR- and extensively drug-resistant (XDR)-TB emergence and spread is largely driven by mismanagement of misadventures in diagnosis, treatment and control of $\mathrm{TB}$, which is compounded by inadequacy of necessary human and financial resources at different levels $[3,4]$.

In the past 24 months the WHO has published two core documents focusing on the importance of the correct case-management of TB: the "End tuberculosis strategy" and the "Framework towards TB elimination in low incidence countries" $[3,5,6]$. Both documents emphasise the importance of prompt diagnosis and effective treatment of newly diagnosed TB cases.

Although there are recently published international standards for TB care and an European adaptation [7-9], and the WHO is currently updating its treatment guidelines [10], major international scientific societies have a critical role to play in the development and implementation of case management guidelines for TB due to their wide membership and access to considerable clinical experience of utilising recommendations. Consequently, the American Thoracic Society (ATS) and the European Respiratory Society (ERS) have developed TB guidelines focused predominantly on TB care in settings without significant resource limitations [11, 12], which have now been updated. Using GRADE (Grading of Recommendations, 


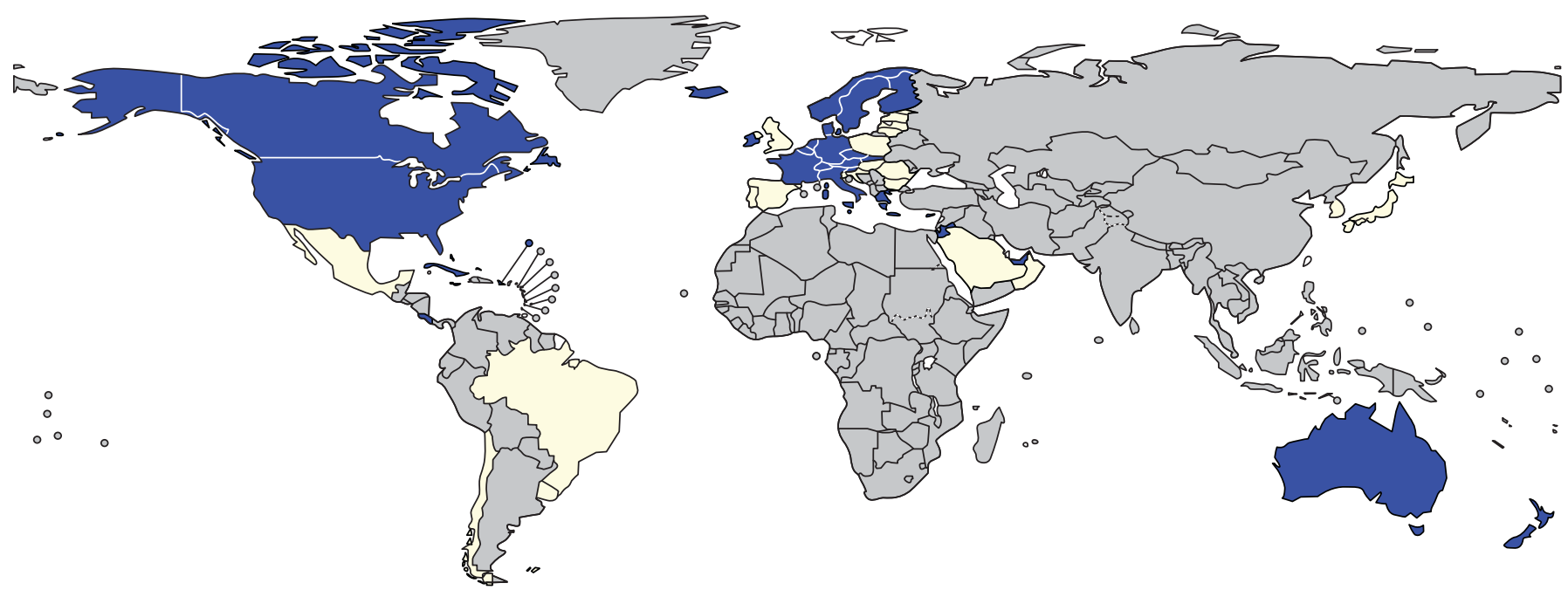

FIGURE 1 Countries with a low incidence $(<10$ cases per 100000 population) of tuberculosis (TB) are shown in blue (2013 data). Estimated incidence 155000 new TB cases per year; 131000 notified cases per year (50000 women, 5000 children); 10000 TB deaths per year (30 deaths per day); 4000 estimated new TB/HIV cases per year; and 567 notified multidrug-resistant TB cases per year.

Assessment, Development and Evaluation) methodology, the ATS, Centers for Disease Control and Prevention (CDC) and Infectious Diseases Society of America (IDSA) jointly sponsored the development of new treatment of drug-susceptible TB clinical practice guidelines, which have been subsequently endorsed by both the ERS and the US National Tuberculosis Controllers Association, and were published in Clinical Infectious Diseases [13].

As before, these guidelines are aimed at providing recommendations on the clinical and public health management of TB in adults and children in settings where diagnostic tests and drugs are available on a routine basis and without restrictions.

\section{Process and methods}

A selected panel of experts, managed for pertinent conflicts of interest according to strict criteria set by the participating Societies, with the necessary competencies, skills and perspectives (pulmonary medicine, infectious diseases, pharmacokinetics, paediatrics, primary care, public health and systematic review methodology) participated as part of the writing committee.

Nine PICO (population, intervention, comparators, outcomes) questions viewed by the writing committee as key clinical questions in the management of active TB and their associated recommendations were developed based on the evidence that was appraised using GRADE [14, 15], and are summarised in table 1. This editorial provides a brief summary of the panel's recommendations; additional important information providing context and references for each recommendation, as well as detailed guidance on the management of TB in special populations, treatment of TB in the presence of HIV infection, TB in children, TB during pregnancy and breastfeeding, and extrapulmonary TB among other clinical situations is available in the full version of the guidelines [13]. Additional detailed guidance on the practical aspects of anti-TB treatment, drug-drug interactions, therapeutic drug monitoring (TDM) and management of adverse events is also available in the full version of the guideline.

What are the principles of anti-TB chemotherapy?

Anti-TB treatment aims to cure the patient, prevent complications and death, avoid relapses, reduce the transmission potential to susceptible individuals, and limit the emergence and spread of drug-resistant strains. For all these reasons, the therapeutic approach to TB requires the use of multiple drugs [12].

A key responsibility of clinicians is making the decision to initiate appropriate treatment for TB. Clinicians decide to start anti-TB chemotherapy based on a variety of data, including clinical, radiographic, laboratory, patient and public health criteria. Commonly, empirical treatment is initiated prior to having definitive confirmation of M. tuberculosis, so as to minimise morbidity and to halt further transmission in the community. Today, fortunately, molecular tests offer rapid diagnosis before culture results are available [16].

Once initiated, treatment success depends upon many factors and an increased risk of relapse has been described among patients with extensive disease (i.e. cavitations or extensive infiltrates on chest radiograph) [17-21] and/or slow with a response to treatment (i.e. culture conversion at 2 or 3 months) [18, 22-24]. 
Here we summarise the rationale and recommendations for the different PICO questions.

\section{Case management interventions (PICO question 1)}

The evidence supports the use of case management strategies in the treatment of TB. In order to ensure patient adherence and maximise the potential for treatment success it is recommended to assign a public health nurse and/or a treatment supporter [13], with whom an individualised "case management plan" is designed, according to a patient-centred approach, as recommended by the International Standards for Tuberculosis Care document [7-9]. This is based on the following elements: 1) educating the patient about the different aspects of treatment and potential adverse events; 2) discussing treatment monitoring procedures; and 3) fostering infection control measures, using simple terms and cultural mediators if necessary (table 1).

\section{Directly observed therapy (PICO question 2)}

The evidence supports the use of directly observed therapy (DOT) in the treatment of TB. Numerous systematic reviews have been conducted to compare outcomes between self-administered therapy (SAT) and DOT (the practice of observing the patient swallow their anti-TB drugs). However, DOT is a part of a multifaceted public health intervention and as such is not amenable to conventional clinical trial approaches assessing benefits/risks. The systematic review conducted to obtain evidence in support of the ERS-endorsed ATS/CDC/IDSA practice guideline did not find any differences between SAT and DOT when assessing mortality, treatment completion and relapse; however, DOT was significantly associated with improved treatment success (the sum of patients cured and patients completing treatment) and with increased sputum smear conversion during treatment, as compared to SAT. As such, these and other international guidelines support the use of DOT, provided in a patient-centred approach, as one component of TB case management [7-10].

\section{The administration schedule of preferred treatment regimens for drug-susceptible TB (PICO questions 3 and 4)}

The preferred regimen for treating adults with $\mathrm{TB}$ caused by strains known or suspected to be drug-susceptible consists of an intensive phase of 2 months (isoniazid (INH), rifampicin (RIF), pyrazinamide (PZA) and ethambutol (EMB)) followed by a continuation phase of 4 months (INH and RIF) [25-27]. The use of four drugs during the intensive phase of treatment ensures its effectiveness in case of INH mono-resistance [13].

If drug susceptibility test (DST) results are known and the patient's isolate is susceptible to both INH and RIF, EMB is not essential and can be discontinued; in this case the intensive phase is composed of INH, RIF and PZA. Pyridoxine (vitamin B6) is given with INH to patients at risk of neuropathy (e.g. pregnant women, breastfeeding infants, individuals co-infected with HIV, the elderly, and patients with diabetes, alcoholism, malnutrition or chronic renal failure).

The recommended frequency of treatment administration is once daily for both the intensive and continuation phases (see PICO questions 3 and 4). However, some experts believe that 5 days-a-week drug administration by DOT is an acceptable alternative to 7 days-a-week. Other alternative regimens that are variations of the preferred regimen, which may be acceptable in certain clinical and/or public health situations, are available in the full text version of the guideline [13].

During treatment, a sputum specimen for direct smear and culture examination is recommended at monthly intervals until two consecutive specimens are negative on culture. As culture status on completion of the intensive phase of treatment ( 2 months) has been shown to correlate with the likelihood of relapse after completion of treatment for pulmonary TB, culture conversion needs to be assessed at the end of 2 months of treatment in new cases [21, 28-30]. Cavitation on the initial chest radiograph has also been shown to be a risk factor for relapse [21, 31]. In patients with cavitation at baseline failing to convert culture after the intensive phase of treatment, rates of relapse have been shown to be higher than among patients with neither factor (20\% versus $2 \%[21,29])$, and based on expert opinion, the extension of the continuation phase with INH and RIF for an additional 3 months (i.e. a continuation phase of 7 months, corresponding to a total of 9 months of therapy) is an option left to the physician in discussion with the patient. Additional factors to be considered in deciding to prolong treatment in patients with either cavitation or a positive culture at 2 months (but not both) might include being underweight (>10\%), being an active smoker, having diabetes, HIV infection or another immunosuppressing condition, or having extensive disease on chest radiography [30, 32-36].

\section{Treatment in special situations}

Detailed recommendations on the management of TB in special situations are available in the full text version of this guideline [13]. Five PICO questions with summary recommendations pertinent to the 
TABLE 1 Nine PICO (population, intervention, comparators, outcomes) questions viewed as key clinical questions in the management of active tuberculosis (TB) and their associated recommendations

\section{PICO question}

1 Does adding case management ${ }^{\#}$ interventions to curative therapy improve outcomes compared with curative therapy alone among patients with TB?

2 Does SAT have similar outcomes compared with DOT in patients with various forms of TB?

3 Does intermittent dosing in the intensive phase have similar outcomes compared with daily dosing in the intensive phase for treatment of drug-susceptible pulmonary TB?
4 Does intermittent dosing in the continuation phase have similar outcomes compared with daily dosing in the continuation phase in patients with drug-susceptible pulmonary TB?

\section{Recommendation(s)}

We suggest using case management interventions during treatment of patients with TB

We suggest using DOT rather than SAT for routine treatment of patients with all forms of TB

We recommend the use of daily rather than intermittent dosing in the intensive phase of therapy for drug-susceptible pulmonary TB

Use of three times weekly therapy in the intensive phase (with or without an initial 2 weeks of daily therapyl may be considered in patients who are not HIV-infected and are also at low risk of relapse I pulmonary TB caused by drug-susceptible organisms, which at the start of treatment is non-cavitary and/or smear negative)

In situations where daily or three times weekly DOT therapy is difficult to achieve, use of twice weekly therapy after an initial 2 weeks of daily therapy may be considered for patients who are not HIV-infected and are also at low risk of relapse I pulmonary TB caused by drug-susceptible organisms, which at the start of treatment is non-cavitary and/or smear negative]

We recommend the use of daily or three times weekly dosing in the continuation phase of therapy for drug-susceptible pulmonary TB

If intermittent therapy is to be administered in the continuation phase, then we suggest use of three times weekly instead of twice weekly therapy

We recommend against use of once weekly therapy with INH $900 \mathrm{mg}$ and RPT $600 \mathrm{mg}$ in the continuation phase

For HIV-infected patients receiving antiretroviral therapy, we suggest using the standard 6-month daily regimen consisting of an intensive phase of 2 months of INH, RIF, PZA and EMB followed by a continuation phase of 4 months of INH and RIF for the treatment of drug-susceptible pulmonary TB

In uncommon situations in which HIV-infected patients do not receive antiretroviral therapy during TB treatment, we suggest extending the continuation phase with INH and RIF for an additional 3 months (i.e. a continuation phase of 7 months in duration, corresponding to a total of 9 months of therapyl for treatment of drug-susceptible pulmonary TB

\section{Comments}

Conditional recommendation/very low confidence in the effects

Conditional recommendation/low confidence in the effects

Strong recommendation/moderate confidence in the effects

Conditional recommendation/low confidence in the effects

Conditional recommendation/very low confidence in the effects

Note: if doses are missed in a regimen using twice weekly dosing then therapy is equivalent to once weekly, which is inferior (see PICO question 4)

Strong recommendation/moderate confidence in the effects

Conditional recommendation/low confidence in the effects

This recommendation allows for the possibility of some doses being missed; with twice weekly therapy, if doses are missed then therapy is equivalent to once weekly, which is inferior

Strong recommendation/high confidence in the effects

In uncommon situations where more than once-weekly DOT is difficult to achieve, once weekly continuation phase therapy with INH 900 mg plus RPT 600 mg may be considered for use only in HIV-negative persons without cavitation on chest radiography

Conditional recommendation/very low confidence in the effects

Conditional recommendation/very low confidence in the effects 
TABLE 1 Continued

PICO question

6 Does initiation of antiretroviral therapy during TB treatment compared with at the end of TB treatment improve outcomes among TB patients co-infected with HIV?

7 Does the use of adjuvant corticosteroids in tuberculous pericarditis provide mortality and morbidity benefits?

8 Does the use of adjuvant corticosteroids in tuberculous meningitis provide mortality and morbidity benefits?

9 Does a shorter duration of treatment have similar outcomes compared with the standard 6-month treatment duration among HIV-negative patients with paucibacillary TB (i.e. smear negative, culture negative)?

\section{Recommendation(s)}

We recommend initiating antiretroviral therapy during TB treatment

Antiretroviral therapy should ideally be initiated within the first 2 weeks of TB treatment for patients with CD4 cell counts $<50$ per $\mathrm{mm}^{3}$ and within 8-12 weeks of TB treatment initiation for patients with CD4 cell counts $\geqslant 50$ per $\mathrm{mm}^{3}$

We suggest initial adjunctive corticosteroid therapy not be routinely used in patients with tuberculous pericarditis

We recommend initial adjunctive corticosteroid therapy with dexamethasone given for 6 weeks for patients with tuberculous meningitis

We suggest that a 4-month treatment regimen is adequate for treatment of HIV-negative adult patients with AFB smear- and culture-negative pulmonary TB

\section{Comments}

Strong recommendation/high confidence in the effects

Note: an exception is patients with HIV infection and tuberculous meningitis

Conditional recommendation/very low confidence in the effects

Strong recommendation/moderate confidence in the effects

Conditional recommendation/very low confidence in the effects

SAT: self-administered therapy; DOT: directly observed therapy; INH: isoniazid; RPT: rifapentin; RIF: rifampicin; PZA: pyrazinamide, EMB: ethambutol; AFB: acid-fast bacilli. \#: case management: patient education/counselling, field/home visits, integration/coordination of care with specialists and medical home, patient reminders, incentives/enablers.

management of $\mathrm{TB}$ in $\mathrm{HIV}$-infected patients, steroid use in pericardial or meningeal tuberculosis, and culture-negative TB are summarised below and in table 1.

\section{HIV infection}

Detailed guidance on the management of TB in HIV-infected patients is provided in the new guidelines, including recommendations on the optimal initiation of antiretroviral therapy (ART), the management of potential drug-drug interactions, especially between rifamycins and ART, and paradoxical reactions among other complexities involved in management of HIV/TB. Several key features are summarised here.

Based on data that show significant reductions in mortality and AIDS-defining illnesses, patients with HIV infection and TB should receive ART in conjunction with daily anti-TB drugs. For HIV-infected patients receiving ART, the standard 6-month daily anti-TB regimen is recommended.

In the uncommon situation in which an HIV-infected patient does not receive ART during anti-TB treatment, the new ATS/CDC/IDSA guidelines suggest extending the continuation phase (INH and RIF) for an additional 3 months (i.e. a continuation phase of 7 months in duration, corresponding to a total of 9 months) for treatment of drug-susceptible pulmonary TB (PICO question 5; table 1).

As high rates of relapse and the emergence of drug resistance has been associated with the use of intermittent regimens, resulting in low serum concentrations of key component drugs in the setting of a low CD4 lymphocyte count $\left(<100\right.$ cells per $\left.\mathrm{mm}^{3}\right)$, based on systematic reviews treatment of HIV-related TB should be administered daily in both the intensive and continuation phases.

On the basis of systematic reviews and meta-analysis, high quality evidence exists showing that benefits outweigh harms; the guidelines recommend that patients with TB and HIV co-infection receive ART during anti-TB treatment. ART should ideally be started within 2 weeks for those patients with a CD4 count $<50$ cells per $\mathrm{mm}^{3}$ and by $8-12$ weeks for those with a CD4 count $\geqslant 50$ cells per $\mathrm{mm}^{3}$ (see PICO question 6). However, in HIV-infected patients with TB meningitis, ART is not initiated in the first 8 weeks of anti-TB therapy due to an association with increased rates of adverse events and higher mortality [37]. The concurrent administration of ART and rifamycins is a major treatment challenge, and details on the co-administration of these medications, including the use of rifabutin (RFB), are available in the full-text version of the guidelines [13].

Patients with TB and HIV co-infection are at increased risk of developing paradoxical worsening of symptoms, signs or clinical manifestations of TB after beginning anti-TB and ART, known as immune reconstitution inflammatory syndrome (IRIS). IRIS is more common in patients with earlier ART 
initiation and $\mathrm{CD}^{+}$cell counts $<50$ cells per $\mathrm{mm}^{3}$ [38]. IRIS may include high fever, worsening respiratory symptoms, inflammation and increased size of involved lymph nodes, new lymphadenopathy, expanding central nervous system lesions, worsening of pulmonary parenchymal infiltrations, new or increasing pleural effusions, and development of intra-abdominal or retroperitoneal abscesses [39].

Management of IRIS is symptomatic. Based on expert opinion, for most patients with mild IRIS, anti-TB and antiretroviral therapies can be continued adding anti-inflammatory drugs such as ibuprofen. For patients with worsening pleural effusions or abscesses, drainage is indicated. For more severe cases of IRIS, corticosteroid treatment is effective. In a trial of prednisone for patients with moderate IRIS, prednisone $1.25 \mathrm{mg} \cdot \mathrm{kg}^{-1}$ per day significantly reduced the need for hospitalisation or surgical procedures [40]. For patients developing IRIS, prednisone may be administered at a dose of $1.25 \mathrm{mg} \cdot \mathrm{kg}^{-1}$ per day (50-80 mg per day) for 2-4 weeks, with tapering over a period of 6-12 weeks or longer.

Co-trimoxazole (trimethoprim-sulfamethoxazole) prophylaxis has been shown to reduce morbidity and mortality in HIV-co-infected patients with newly diagnosed TB [41-43]. The WHO recommends co-trimoxazole for all HIV-infected individuals with active TB regardless of their CD4 cell count [44], while in high-income countries co-trimoxazole is primarily used in HIV-infected patients with CD4 counts $<200$ cells per $\mathrm{mm}^{3}$ [45]. The use of ART during anti-TB treatment in HIV co-infected patients also reduces mortality rates significantly while decreasing the risk of developing AIDS-related conditions.

\section{TB pericarditis}

Based on systematic reviews conducted in support of the guidelines, greatly informed by a recent placebo-controlled randomised clinical trial with 1400 participants [46], adjunctive corticosteroids should not be used routinely in the treatment of patients with pericardial TB (PICO question 7) [46-50]. However, selective use of corticosteroids in patients who are at the highest risk for inflammatory complications might be appropriate.

\section{TB meningitis}

Treatment for TB meningitis includes INH, RIF, PZA and EMB in the initial 2-month phase. In the continuation phase of treatment, for meningitis due to strains known or presumed to be drug-susceptible, INH and RIF should be continued for an additional 7-10 months, although the optimal duration of chemotherapy is not defined ( 12 months in the UK). Expert opinion suggest that repeated lumbar punctures may be used to monitor changes in cerebrospinal fluid cell count, glucose and protein, especially in the early phases of treatment. In children with TB meningitis, the regimen recommended consists of INH, RIF, PZA and ethionamide, if possible, or an aminoglycoside, for 2 months followed by 7-10 months of INH and RIF [51]. For adults, based on expert opinion, the guidelines recommend using $\mathrm{EMB}$ as the fourth drug in the regimen.

The role of adjunctive corticosteroid therapy in the treatment of TB meningitis has been investigated by numerous studies [52-64], and the updated systematic review conducted in support of the guidelines showed a mortality benefit from the use of adjuvant corticosteroids. Therefore, the guidelines recommend adjunctive corticosteroid therapy with dexamethasone or prednisolone tapered over 6-8 weeks for patients with TB meningitis (PICO question 8).

\section{Culture-negative pulmonary TB in adults}

Based on a systematic review conducted in support of the guideline, a 4-month treatment regimen was shown to be adequate for sputum smear-negative, culture-negative pulmonary TB (PICO question 9). The intensive phase of treatment includes INH, RIF, PZA and EMB daily, and is continued even when the initial bacteriological studies are negative. If all cultures on adequate samples are negative (culture-negative TB) and there is a clinical or radiographic response after 2 months of intensive phase therapy, the continuation phase with INH and RIF may be shortened to 2 months in HIV-negative adults (but the confidence in the effects for this recommendation is very low). Alternatively, if there is concern about the adequacy of work-up or the accuracy of the microbiological evaluations, a standard 6-month regimen remains preferred $[7,8]$. Importantly, the guidelines note that causes of failure to isolate organisms should be considered and these include the recent use of antibiotics with bactericidal activity against M. tuberculosis (e.g. fluoroquinolones), low bacillary populations, inadequate sputum specimens, temporal variations in the number of expelled bacilli, overgrowth of cultures with other microorganisms, and errors in specimen processing [65]. At a minimum, patients suspected of having pulmonary TB should have two sputum specimens (using sputum induction with hypertonic saline if necessary) for alcohol acid-fast bacilli smears and cultures for mycobacteria or for rapid molecular testing for M.tuberculosis as part of the diagnostic evaluation. Bronchoscopy with bronchoalveolar lavage and/or biopsy have also to be considered before making a presumptive diagnosis of culture-negative TB. 


\section{Conclusions}

The main goals of anti-TB treatment are to cure individual patients and minimise transmission of M. tuberculosis within the community. The standard four-drug regimen (INH, RIF, PZA and EMB) remains the preferred initial treatment for drug-susceptible pulmonary TB. Treatment needs to start even before direct smear microscopy, molecular tests and mycobacterial culture results are known in patients with a high likelihood of having TB and/or who are seriously ill.

Variations of the preferred regimen that are appropriate in certain public health situations or in special clinical situations and additional detailed information on TB treatment are available in the full-text version of the guidelines [13].

\section{Acknowledgements}

The authors wish to thank Rosella Centis and Lia D’Ambrosio, WHO Collaborating Centre for TB and Lung Diseases, Fondazione S. Maugeri, Tradate, Italy for their useful comments and for the editorial support in finalising the manuscript.

\section{References}

1 World Health Organization. Global tuberculosis report 2015. WHO/HTM/TB/2015.22. Geneva, World Health Organization, 2015.

2 World Health Organization. Global tuberculosis report 2013. WHO/HTM/TB/2013.11. Geneva, World Health Organization, 2013.

3 Uplekar M, Weil D, Lonnroth K, et al. WHO's new End TB Strategy. Lancet 385: 1799-1801.

4 Migliori GB, Sotgiu G, D'Ambrosio L, et al. TB and MDR/XDR-TB in European Union and European Economic Area countries: managed or mismanaged? Eur Respir J 2012; 39: 619-625.

5 Sotgiu G, Mauch V, Migliori GB, et al. Evidence-based, agreed-upon health priorities to remedy the tuberculosis patient's economic disaster. Eur Respir J 2014; 43: 1563-1566.

6 Lönnroth K, Migliori GB, Abubakar I, et al. Towards tuberculosis elimination: an action framework for low-incidence countries. Eur Respir J 2015; 45: 928-952.

7 TB CARE I, UNAIDS. International Standards for Tuberculosis Care. 3rd Edn. The Hague, TB CARE I, 2014.

8 Migliori GB, Zellweger JP, Abubakar I, et al. European union standards for tuberculosis care. Eur Respir J 2012; 39: 807-819.

9 van der Werf MJ, Sandgren A, D'Ambrosio L, et al. The European Union standards for tuberculosis care: do they need an update? Eur Respir J 2014; 43: 933-942.

10 World Health Organization. Treatment of Tuberculosis: guidelines. WHO/HTM/TB/2009.420. 4th Edn. Geneva, World Health Organization, 2010.

11 Blumberg HM, Burman WJ, Chaisson RE, et al. American Thoracic Society/Centers for Disease Control and Prevention/Infectious Diseases Society of America. Treatment of tuberculosis. Am J Respir Crit Care Med 2003; 167: 603-662.

12 Migliori GB, Raviglione MC, Schaberg T, et al. Tuberculosis management in Europe. Task Force of the European Respiratory Society (ERS), the World Health Organization (WHO) and the International Union against Tuberculosis and Lung Disease (IUATLD) Europe Region. Eur Respir J 1999; 14: 978-992.

13 Nahid P, Dorman SE, Alipanah N, et al. Official American Thoracic Society/Centers for Disease Control/ Infectious Diseases Society of America Clinical Practice Guidelines: Treatment of Drug-Susceptible Tuberculosis. Clin Infect Dis 2016; in press [DOI: 10.1093/cid/ciw376].

14 Schunemann HJ, Jaeschke R, Cook DJ, et al. An official ATS statement: grading the quality of evidence and strength of recommendations in ATS guidelines and recommendations. Am J Respir Crit Care Med 2006; 174: 605-614.

15 Guyatt GH, Oxman AD, Vist GE, et al. GRADE: an emerging consensus on rating quality of evidence and strength of recommendations. BMJ 2008; 336: 924-926.

16 World Health Organization. WHO treatment guidelines for drug-resistant tuberculosis 2016 update. WHO/HTM/ TB/2016.04. Geneva, World Health Organization, 2016.

17 Gillespie SH, Crook AM, McHugh TD, et al. Four-month moxifloxacin-based regimens for drug-sensitive tuberculosis. N Engl J Med 2014; 371: 1577-1587.

18 Zierski M, Bek E. Side-effects of drug regimens used in short-course chemotherapy for pulmonary tuberculosis. A controlled clinical study. Tubercle 1980; 61: 41-49.

19 Phillips PP, Fielding K, Nunn AJ. An evaluation of culture results during treatment for tuberculosis as surrogate endpoints for treatment failure and relapse. PLoS One 2013; 8: e63840.

20 Aber VR, Nunn AJ. Chimiothérapie de courte duree de la tuberculose. Facteurs de rechute dans la chimiothérapie de courte durée [Short term chemotherapy of tuberculosis. Factors affecting relapse following short term chemotherapy]. Bull Int Union Tuberc 1978; 53: 276-280.

21 Benator D, Bhattacharya $\mathrm{M}$, Bozeman L, et al. Rifapentine and isoniazid once a week versus rifampicin and isoniazid twice a week for treatment of drug-susceptible pulmonary tuberculosis in HIV-negative patients: a randomised clinical trial. Lancet 2002; 360: 528-534.

22 Mitchison D, Davies G. The chemotherapy of tuberculosis: past, present and future. Int J Tuberc Lung Dis 2012; 16: 724-732.

23 Menzies D, Elwood K. Treatment of Tuberculosis Disease. In: Canadian Tuberculosis Standards. 7th Edn. Ottawa, Centre for Communicable Diseases and Infection Control, Public Health Agency of Canada, 2014.

24 Weiner M, Burman W, Vernon A, et al. Low isoniazid concentrations and outcome of tuberculosis treatment with once-weekly isoniazid and rifapentine. Am J Respir Crit Care Med 2003; 167: 1341-1347.

25 Fox W, Ellard GA, Mitchison DA. Studies on the treatment of tuberculosis undertaken by the British Medical Research Council tuberculosis units, 1946-1986, with relevant subsequent publications. Int J Tuberc Lung Dis 1999; 3: Suppl. 2, S231-S279.

26 Menzies D, Benedetti A, Paydar A, et al. Effect of duration and intermittency of rifampin on tuberculosis treatment outcomes: a systematic review and meta-analysis. PLoS Med 2009; 6: e1000146. 

treatment and/or with mono-resistance to isoniazid: a systematic review and meta-analysis. PLoS Med 2009; 6: e1000150.

28 Mitchison DA. Assessment of new sterilizing drugs for treating pulmonary tuberculosis by culture at 2 months. Am Rev Respir Dis 1993; 147: 1062-1063.

29 Jo KW, Yoo JW, Hong Y, et al. Risk factors for 1-year relapse of pulmonary tuberculosis treated with a 6-month daily regimen. Respir Med 2014; 108: 654-659.

30 Horne DJ, Royce SE, Gooze L, et al. Sputum monitoring during tuberculosis treatment for predicting outcome: systematic review and meta-analysis. Lancet Infect Dis 2010; 10: 387-394.

31 Chang KC, Leung CC, Yew WW, et al. A nested case-control study on treatment-related risk factors for early relapse of tuberculosis. Am J Respir Crit Care Med 2004; 170: 1124-1130.

32 Khan A, Sterling TR, Reves R, et al. Lack of weight gain and relapse risk in a large tuberculosis treatment trial. Am J Respir Crit Care Med 2006; 174: 344-348.

33 Baker MA, Harries AD, Jeon CY, et al. The impact of diabetes on tuberculosis treatment outcomes: a systematic review. BMC Med 2011; 9: 81.

34 Wang JY, Lee MC, Shu CC, et al. Optimal duration of anti-TB treatment in patients with diabetes: nine or six months? Chest 2015; 147: 520-528.

35 Leung CC, Yew WW, Chan CK, et al. Smoking adversely affects treatment response, outcome and relapse in tuberculosis. Eur Respir J 2015; 45: 738-745.

36 Ahmad Khan F, Minion J, Al-Motairi A, et al. An updated systematic review and meta-analysis on the treatment of active tuberculosis in patients with HIV infection. Clin Infect Dis 2012; 55: 1154-1163.

37 Torok ME, Yen NT, Chau TT, et al. Timing of initiation of antiretroviral therapy in human immunodeficiency virus (HIV) - associated tuberculous meningitis. Clin Infect Dis 2011; 52: 1374-1383.

38 Luetkemeyer AF, Kendall MA, Nyirenda M, et al. Tuberculosis immune reconstitution inflammatory syndrome in A5221 STRIDE: timing, severity, and implications for HIV-TB programs. J Acquir Immune Defic Syndr 2014; 65: 423-428.

39 Meintjes G, Lawn SD, Scano F, et al. Tuberculosis-associated immune reconstitution inflammatory syndrome: case definitions for use in resource-limited settings. Lancet Infect Dis 2008; 8: 516-523.

40 Meintjes G, Wilkinson RJ, Morroni C, et al. Randomized placebo-controlled trial of prednisone for paradoxical tuberculosis-associated immune reconstitution inflammatory syndrome. AIDS 2010; 24: 2381-2390.

41 Nunn AJ, Mwaba P, Chintu C, et al. Role of co-trimoxazole prophylaxis in reducing mortality in HIV infected adults being treated for tuberculosis: randomised clinical trial. BMJ 2008; 337: a257.

42 Suthar AB, Granich R, Mermin J, et al. Effect of cotrimoxazole on mortality in HIV-infected adults on antiretroviral therapy: a systematic review and meta-analysis. Bull World Health Organ 2012; 90: 128C-138C.

43 Wiktor SZ, Sassan-Morokro M, Grant AD, et al. Efficacy of trimethoprim-sulphamethoxazole prophylaxis to decrease morbidity and mortality in HIV-1-infected patients with tuberculosis in Abidjan, Cote d'Ivoire: a randomised controlled trial. Lancet 1999; 353: 1469-1475.

44 World Health Organization. Guidelines on post-exposure prophylaxis for HIV and the use of co-trimoxazole prophylaxis for HIV-related infections among adults, adolescents and children. Recommendations for a public health approach - December 2014 supplement to the 2013 consolidated guidelines on the use of antiretroviral drugs for treating and preventing HIV infection. Geneva, World Health Organization, 2014.

45 Masur H, Brooks JT, Benson CA, et al. Prevention and treatment of opportunistic infections in HIV-infected adults and adolescents: updated guidelines from the Centers for Disease Control and Prevention, National Institutes of Health, and HIV Medicine Association of the Infectious Diseases Society of America. Clin Infect Dis 2014; 58: 1308-1311.

46 Mayosi BM, Ntsekhe M, Smieja M. Immunotherapy for tuberculous pericarditis. N Engl J Med 2014; $371: 2534$.

47 Strang JI, Kakaza HH, Gibson DG, et al. Controlled trial of prednisolone as adjuvant in treatment of tuberculous constrictive pericarditis in Transkei. Lancet 1987; 2: 1418-1422.

48 Strang JI, Kakaza HH, Gibson DG, et al. Controlled clinical trial of complete open surgical drainage and of prednisolone in treatment of tuberculous pericardial effusion in Transkei. Lancet 1988; 2: 759-764.

49 Hakim JG, Ternouth I, Mushangi E, et al. Double blind randomised placebo controlled trial of adjunctive prednisolone in the treatment of effusive tuberculous pericarditis in HIV seropositive patients. Heart 2000; 84: 183-188.

50 Reuter H, Burgess LJ, Louw VJ, et al. Experience with adjunctive corticosteroids in managing tuberculous pericarditis. Cardiovasc J S Afr 2006; 17: 233-238.

51 Kimberlin DW, Brady MT, Jackson MA, et al. eds. Committee on Infectious Diseases. Red Book: 2015 Report of the Committee on Infectious Diseases. 30th Edn. Elk Grove Village, American Academy of Pediatrics, 2015.

52 Ashby M, Grant H. Tuberculous meningitis treated with cortisone. Lancet 1955; 268: 65-66.

53 O'Toole RD, Thornton GF, Mukherjee MK, et al. Dexamethasone in tuberculous meningitis. Relationship of cerebrospinal fluid effects to therapeutic efficacy. Ann Intern Med 1969; 70: 39-48.

54 Escobar JA, Belsey MA, Duenas A, et al. Mortality from tuberculous meningitis reduced by steroid therapy. Pediatrics 1975; 56: 1050-1055.

55 Girgis NI, Farid Z, Hanna LS, et al. The use of dexamethasone in preventing ocular complications in tuberculous meningitis. Trans R Soc Trop Med Hyg 1983; 77: 658-659.

56 Girgis NI, Farid Z, Kilpatrick ME, et al. Dexamethasone adjunctive treatment for tuberculous meningitis. Pediatr Infect Dis J 1991; 10: 179-183.

57 Kumarvelu S, Prasad K, Khosla A, et al. Randomized controlled trial of dexamethasone in tuberculous meningitis. Tuber Lung Dis 1994; 75: 203-207.

58 Chotmongkol V, Jitpimolmard S, Thavornpitak Y. Corticosteroid in tuberculous meningitis. J Med Assoc Thai 1996; 79: 83-90.

59 Dooley DP, Carpenter JL, Rademacher S. Adjunctive corticosteroid therapy for tuberculosis: a critical reappraisal of the literature. Clin Infect Dis 1997; 25: 872-887.

60 Schoeman JF, Van Zyl LE, Laubscher JA, et al. Effect of corticosteroids on intracranial pressure, computed tomographic findings, and clinical outcome in young children with tuberculous meningitis. Pediatrics 1997; 99: $226-231$. 
61 Thwaites GE, Nguyen DB, Nguyen HD, et al. Dexamethasone for the treatment of tuberculous meningitis in adolescents and adults. N Engl J Med 2004; 351: 1741-1751.

62 Prasad K, Singh MB. Corticosteroids for managing tuberculous meningitis. Cochrane Database Syst Rev 2008; 1: CD002244.

63 Malhotra HS, Garg RK, Singh MK, et al. Corticosteroids (dexamethasone versus intravenous methylprednisolone) in patients with tuberculous meningitis. Ann Trop Med Parasitol 2009; 103: 625-634.

64 Critchley JA, Young F, Orton L, et al. Corticosteroids for prevention of mortality in people with tuberculosis: a systematic review and meta-analysis. Lancet Infect Dis 2013; 13: 223-237.

65 Ho J, Marks GB, Fox GJ. The impact of sputum quality on tuberculosis diagnosis: a systematic review. Int J Tuberc Lung Dis 2015; 19: 537-544. 